\title{
Investigations on mechanical behaviour of dental composites
}

\author{
Nicoleta Ilie $\cdot$ Reinhard Hickel
}

Published online: 29 April 2009

(C) Springer-Verlag 2009

\section{Erratum to: Clin Oral Invest \\ DOI 10.007/s00784-009-0258-4}

The presentation of Table 2 was incorrect.

Under "Material type" the data in the columns 'CS', 'Vol\%', and $\mathrm{Wt} \%$, were interchanged.

The correct version is given below.

Table 2 Flexural strength (FS) and modulus (FM), diametral tensile strength (DTS) and compressive strength (CS)

\begin{tabular}{|c|c|c|c|c|}
\hline Composites & $\mathrm{FS}[\mathrm{MPa}]$ & $\mathrm{FM}[\mathrm{GPa}]$ & DTS [MPa] & $\mathrm{CS}[\mathrm{MPa}]$ \\
\hline \multicolumn{5}{|l|}{ Hybrid composites } \\
\hline $\mathrm{Z} 250$ & $160.8^{\mathrm{i}}(22.2)$ & $10.3^{\operatorname{lm}}(0.6)$ & $33.1^{\text {abcde }}(3.6)$ & $282.9^{\mathrm{k}}(25.6)$ \\
\hline Synergy Duo Shade & $143.5^{\mathrm{hi}}(16.3)$ & $8.7^{\mathrm{hijkl}}(0.6)$ & $28.2^{\mathrm{abc}}(3.7)$ & $272.5^{\mathrm{jk}}(27.8)$ \\
\hline Ecusit Composite & $140.7^{\text {ghi }}(15.5)$ & $9.6^{\mathrm{ijkl}}(0.5)$ & $30.3^{\mathrm{abc}}(5.1)$ & $263.5^{\text {hijk }}(19.5)$ \\
\hline Tetric & $139.9^{\text {ghi }}(15.0)$ & $9.9^{\mathrm{jklm}}(0.7)$ & $30.6^{\mathrm{abcd}}(3.9)$ & $261.2^{\text {hijk }}(27.8)$ \\
\hline TPH Spectrum & $138.6^{\mathrm{ghi}}(15.6)$ & $8.8^{\text {hijkl }}(0.3)$ & $42.1^{\operatorname{def}}(6.9)$ & $261.2^{\text {hijk }}(28.0)$ \\
\hline Enamel plus HFO & $138.4^{\text {ghi }}(10.2)$ & $8.5^{\text {ghijk }}(1.7)$ & $33.2^{\text {abcde }}(4.0)$ & $199.1^{\text {efghi }}(44.9)$ \\
\hline Adamant Cavifil & $135.9^{\text {fghi }}(18.0)$ & $7.7^{\mathrm{efgh}}(1.2)$ & $47.7^{\mathrm{f}}(15.9)$ & $206.1^{\text {efghij }}(58.7)$ \\
\hline $\mathrm{Z} 100$ & $134.5^{\text {gghi }}(18.0)$ & $11.3^{\mathrm{m}}(0.5)$ & $33.5^{\text {abcde }}(4.8)$ & $256.6^{\text {hijk }}(36.2)$ \\
\hline Prodigy & $126.6^{\text {efgh }}(20.9)$ & $7.8^{\mathrm{fgh}}(0.3)$ & $38.5^{\text {bcdef }}(5.5)$ & $253.6^{\mathrm{hijk}}(26.9)$ \\
\hline Arabesk Top & $123.1^{\mathrm{defgh}}(23.3)$ & $8.1^{\text {fghi }}(0.6)$ & $26.9^{\mathrm{ab}}(4.7)$ & $248.8^{\text {ghijk }}(48.4)$ \\
\hline Herculite XRV & $121.8^{\text {cdefgh }}(12.4)$ & $8.5^{\text {ghij }}(0.6)$ & $31.8^{\mathrm{abcd}}(2.3)$ & $251.4^{\text {hijk }}(33.0)$ \\
\hline Ariston $\mathrm{pHc}$ & $119.3^{\text {bcdefgh }}(11.1)$ & $7.3^{\text {efgh }}(0.9)$ & $44.2^{\mathrm{ef}}(5.6)$ & $179.3^{\text {cdefg }}(62.0)$ \\
\hline Brillant & $119.2^{\text {bcdefgh }}(13.9)$ & $7.7^{\text {efgh }}(0.6)$ & $31.0^{\text {abcd }}(3.7)$ & $218.0^{\text {fghijk }}(33.9)$ \\
\hline Pertac II & $117.5^{\text {bcdefgh }}(12.6)$ & $8.7^{\text {hijkl }}(0.3)$ & $30.4^{\mathrm{abcd}}(3.3)$ & $242.2^{\text {ghijk }}(38.0)$ \\
\hline Tetric Ceram & $116.9^{\text {bcdefgh }}(9.2)$ & $8.1^{\text {fghi }}(0.4)$ & $39.2^{\text {cdef }}(3.7)$ & $248.0^{\text {ghijk }}(24.8)$ \\
\hline Superlux Universalhybrid & $113.9^{\text {bcdefg }}(9.2)$ & $6.9^{\mathrm{efg}}(0.7)$ & $32.0^{\mathrm{abcd}}(7.8)$ & $267.7^{\mathrm{ijk}}(53.4)$ \\
\hline EcuSphere Carat & $112.8^{\text {bcdefg }}(17.6)$ & $6.6^{\operatorname{def}}(0.8)$ & $34.7^{\text {bcde }}(4.4)$ & $252.3^{\text {hijk }}(27.6)$ \\
\hline Venus & $108.6^{\text {bcdef }}(14.7)$ & $2.9^{\mathrm{a}}(0.6)$ & $31.0^{\mathrm{abcd}}(5.0)$ & $156.8^{\text {bcdef }}(35.6)$ \\
\hline Hermes & $108.5^{\text {bcdef }}(24.0)$ & $7.5^{\text {efgh }}(1.4)$ & $31.6^{\mathrm{abcd}}(7.2)$ & $129.1^{\text {abcd }}(43.3)$ \\
\hline
\end{tabular}

The online version of the original article can be found at http://dx.doi. org/10.1007/s00784-009-0258-4.

N. Ilie $(\bowtie) \cdot$ R. Hickel

Department of Restorative Dentistry, Dental School,

Ludwig-Maximilians-University,

Goethestr. 70,

80336 Munich, Germany

e-mail: nicoleta.ilie@dent.med.uni-muenchen.de 
Table 2 (continued)

\begin{tabular}{|c|c|c|c|c|}
\hline Composites & $\mathrm{FS}[\mathrm{MPa}]$ & $\mathrm{FM}[\mathrm{GPa}]$ & DTS [MPa] & $\mathrm{CS}[\mathrm{MPa}]$ \\
\hline Esthet-X & $106.8^{\text {bcdef }}(13.6)$ & $7.8^{\text {fgh }}(0.6)$ & $29.3^{\mathrm{abc}}(3.7)$ & $272.4^{\mathrm{jk}}(13.2)$ \\
\hline Point 4 & $104.2^{\text {bcde }}(14.0)$ & $5.7^{\mathrm{cd}}(1.5)$ & $27.0^{\mathrm{ab}}(11.5)$ & $150.2^{\text {bcdef }}(35.2)$ \\
\hline Reference & $102.7^{\text {abcde }}(8.5)$ & $10.1^{\mathrm{klm}}(1.8)$ & $37.5^{\text {bcdef }}(6.7)$ & $284.4^{\mathrm{k}}(35.0)$ \\
\hline Charisma & $102.4^{\text {abcde }}(11.1)$ & $7.1^{\mathrm{efg}}(0.5)$ & $27.3^{\mathrm{ab}}(3.0)$ & $263.3^{\mathrm{hijk}}(15.3)$ \\
\hline Beautifil & $98.4^{\text {abcde }}(16.6)$ & $5.2^{\mathrm{cd}}(1.0)$ & $29.0^{\mathrm{abc}}(7.9)$ & $121.6^{\mathrm{abc}}(27.1)$ \\
\hline Clearfil ST & $97.5^{\text {abcde }}(16.8)$ & $6.1^{\text {cde }}(1.0)$ & $27.6^{\mathrm{abc}}(5.0)$ & $150.8^{\text {bcdef }}(45.1)$ \\
\hline Miris & $94.9^{\mathrm{abcd}}(16.6)$ & $4.7^{\mathrm{bc}}(1.2)$ & $22.4^{\mathrm{a}}(7.9)$ & $70.8^{\mathrm{a}}(23.5)$ \\
\hline InTenS & $93.1^{\mathrm{abc}}(9.8)$ & $4.8^{\mathrm{bc}}(0.5)$ & $35.9^{\text {bcdef }}(6.3)$ & $195.3^{\text {defgh }}(37.6)$ \\
\hline CapoCom.4 & $91.8^{\mathrm{ab}}(19.0)$ & $3.4^{\mathrm{ab}}(0.8)$ & $30.3^{\mathrm{abc}}(4.9)$ & $100.4^{\mathrm{ab}}(17.5)$ \\
\hline ELS Extra low shrinkage & $73.6^{\mathrm{a}}(19.0)$ & $2.4^{\mathrm{a}}(0.8)$ & $26.9^{\mathrm{ab}}(6.5)$ & $138.3^{\text {abcde }}(56.6)$ \\
\hline \multicolumn{5}{|l|}{ Nano-hybrid composites } \\
\hline Filtek Supreme Enamel & $125.5^{\mathrm{d}}(13.1)$ & $5.4^{\mathrm{bc}}(0.6)$ & $39.2^{\mathrm{abc}}(8.3)$ & $267.4^{\mathrm{b}}(38.3)$ \\
\hline Filtek Supreme Dentin & $122.1^{\mathrm{cd}}(14.4)$ & $5.2^{\mathrm{ab}}(0.5)$ & $41.8^{\mathrm{abc}}(7.8)$ & $103.7^{\mathrm{a}}(20.9)$ \\
\hline Filtek Supreme XT & $108.6^{\mathrm{bcd}}(19.0)$ & $6.1^{\mathrm{c}}(1.1)$ & $35.8^{\mathrm{ab}}(7.6)$ & $134.3^{\mathrm{a}}(46.7)$ \\
\hline CeramX Duo & $100.9^{\mathrm{abc}}(17.9)$ & $4.5^{\mathrm{ab}}(0.5)$ & $32.0^{\mathrm{a}}(4.9)$ & $214.9^{\mathrm{b}}(25.6)$ \\
\hline Tetric Evo Ceram & $96.0^{\mathrm{ab}}(14.3)$ & $5.3^{\mathrm{bc}}(0.5)$ & $38.5^{\mathrm{abc}}(5.2)$ & $219.7^{\mathrm{b}}(46.9)$ \\
\hline CeramX Mono & $95.0^{\mathrm{ab}}(13.3)$ & $4.3^{\mathrm{a}}(0.4)$ & $39.6^{\mathrm{abc}}(7.9)$ & $240.7^{\mathrm{b}}(25.8)$ \\
\hline Premise Enamel & $82.2^{\mathrm{a}}(8.1)$ & $5.0^{\mathrm{ab}}(0.5)$ & $45.1^{\mathrm{bc}}(4.5)$ & $242.8^{\mathrm{b}}(36.1)$ \\
\hline \multicolumn{5}{|l|}{ Micro-filled composites } \\
\hline Heliomolar radiopaque & $87.6^{\mathrm{c}}(3.0)$ & $4.1^{\mathrm{b}}(0.2)$ & $25.1^{\mathrm{ab}}(2.4)$ & $231.3^{\mathrm{a}}(22.9)$ \\
\hline Durafill & $76.4^{\mathrm{b}}(9.2)$ & $3.0^{\mathrm{a}}(0.2)$ & $21.5^{\mathrm{a}}(2.4)$ & $289.8^{\mathrm{b}}(33.1)$ \\
\hline EcuSphere Shine & $68.4^{\mathrm{a}}(6.1)$ & $3.3^{\mathrm{a}}(0.3)$ & $27.2^{\mathrm{b}}(4.5)$ & $219.2^{\mathrm{a}}(22.2)$ \\
\hline Silux Plus & $62.8^{\mathrm{a}}(3.4)$ & $5.0^{\mathrm{c}}(0.3)$ & $22.4^{\mathrm{a}}(2.1)$ & $254.3^{\mathrm{ab}}(30.4)$ \\
\hline \multicolumn{5}{|l|}{ Packable composites } \\
\hline Filtek P60 & $136.2^{\mathrm{d}}(9.9)$ & $10.2^{\mathrm{c}}(0.5)$ & $37.3^{\mathrm{cd}}(6.4)$ & $273.7^{\mathrm{c}}(43.8)$ \\
\hline Alert & $124.7^{\mathrm{cd}}(22.1)$ & $12.5^{\mathrm{d}}(2.1)$ & $46.8^{\mathrm{e}}(3.1)$ & $275.6^{\mathrm{c}}(22.9)$ \\
\hline Synergy Compact & $124.1^{\mathrm{cd}}(11.8)$ & $8.2^{\mathrm{bc}}(0.6)$ & $22.9^{\mathrm{a}}(4.5)$ & $195.4^{\mathrm{ab}}(34.8)$ \\
\hline Surefil & $110.5^{\text {bcd }}(24.3)$ & $7.4^{\mathrm{b}}(2.0)$ & $31.9^{\mathrm{bc}}(7.8)$ & $180.9^{\mathrm{a}}(82.2)$ \\
\hline Prodigy Condensable & $102.4^{\mathrm{bc}}(32.3)$ & $9.1^{\mathrm{bc}}(1.4)$ & $32.2^{\mathrm{bc}}(5.7)$ & $141.5^{\mathrm{a}}(25.7)$ \\
\hline Solitaire 2 & $100.2^{\mathrm{bc}}(9.4)$ & $7.7^{\mathrm{b}}(0.5)$ & $32.0^{\mathrm{bc}}(2.9)$ & $285.0^{\mathrm{c}}(26.7)$ \\
\hline Quix Fil & $99.5^{\mathrm{bc}}(29.4)$ & $7.6^{\mathrm{b}}(1.2)$ & $42.7^{\mathrm{de}}(7.8)$ & $212.7^{\mathrm{abc}}(30.3)$ \\
\hline Solitaire 1 & $82.3^{\mathrm{ab}}(9.9)$ & $4.3^{\mathrm{a}}(0.3)$ & $26.4^{\mathrm{ab}}(2.3)$ & $257.2^{\mathrm{bc}}(25.6)$ \\
\hline Tetric Condensable & $65.4^{\mathrm{a}}(17.3)$ & $8.7^{\mathrm{bc}}(2.0)$ & $38.9^{\text {cde }}(4.7)$ & $171.0^{\mathrm{a}}(39.7)$ \\
\hline \multicolumn{5}{|l|}{ Ormocer-based composites } \\
\hline Definite & $120.5(3.2)$ & $7.1(0.6)$ & $26.9(3.5)$ & $223.7(32.2)$ \\
\hline Admira & $88.2(19.7)$ & $7.9(3.5)$ & $43.4(5.2)$ & $208.4(31.0)$ \\
\hline \multicolumn{5}{|l|}{ Compomeres } \\
\hline Luxat & $131.6^{\mathrm{d}}(24.2)$ & $12.4^{\mathrm{d}}(0.6)$ & $48.9^{\mathrm{d}}(5.4)$ & $256.6^{\mathrm{b}}(19.4)$ \\
\hline Hytac & $123.5^{\mathrm{cd}}(16.7)$ & $11.7^{\mathrm{d}}(0.7)$ & $45.2^{\mathrm{d}}(5.4)$ & $227.7^{\mathrm{b}}(14.5)$ \\
\hline Compoglass $\mathrm{F}$ & $104.0^{\text {bcd }}(21.7)$ & $8.8^{\mathrm{bc}}(0.6)$ & $29.7^{\mathrm{abc}}(3.3)$ & $201.4^{\mathrm{a}}(48.3)$ \\
\hline Dyract & $101.0^{\mathrm{bc}}(22.9)$ & $7.3^{\mathrm{a}}(0.8)$ & $33.1^{\mathrm{bc}}(4.1)$ & $247.8^{\mathrm{ab}}(22.7)$ \\
\hline Glasiosite & $100.1^{\mathrm{bc}}(15.5)$ & $9.0^{\mathrm{c}}(0.5)$ & $36.4^{\mathrm{c}}(5.7)$ & $237.6^{\mathrm{ab}}(32.6)$ \\
\hline Dyract AP & $84.9^{\mathrm{b}}(16.0)$ & $7.7^{\mathrm{ab}}(0.6)$ & $25.4^{\mathrm{a}}(2.2)$ & $201.5^{\mathrm{a}}(19.4)$ \\
\hline Elan & $81.5^{\mathrm{b}}(18.2)$ & $7.9^{\mathrm{abc}}(1.0)$ & $25.5^{\mathrm{a}}(4.1)$ & $256.4^{\mathrm{ab}}(45.2)$ \\
\hline F2000 Rasant & $31.0^{\mathrm{a}}(9.5)$ & $8.4^{\mathrm{bc}}(0.8)$ & $26.8^{\mathrm{ab}}(2.8)$ & $218.5^{\mathrm{ab}}(15.4)$ \\
\hline
\end{tabular}


Table 2 (continued)

\begin{tabular}{|c|c|c|c|c|c|c|}
\hline Composites & $\mathrm{FS}[\mathrm{MPa}]$ & $\mathrm{FM}[\mathrm{GPa}]$ & DTS [MPa] & $\mathrm{CS}[\mathrm{MPa}]$ & & \\
\hline \multicolumn{7}{|l|}{ Flowable composites } \\
\hline FLOWline & $133.0^{\mathrm{g}}(13.9)$ & $5.1^{\text {bcde }}(0.4)$ & $53.6^{\mathrm{d}}(6.5)$ & $286.3^{\mathrm{bc}}(29.4)$ & & \\
\hline Arabesk Flow & $131.7^{\mathrm{g}}(22.0)$ & $6.4^{\mathrm{e}}(0.7)$ & $38.4^{\mathrm{abc}}(7.0)$ & $266.8^{\mathrm{bc}}(36.0)$ & & \\
\hline Tetric Flow & $114.7^{\mathrm{efg}}(13.1)$ & $4.8^{\mathrm{bcd}}(1.4)$ & $38.6^{\mathrm{abc}}(4.9)$ & $257.3^{\mathrm{abc}}(48.6)$ & & \\
\hline Palfique Estelite Low Flow & $105.1^{\mathrm{def}}(15.3)$ & $2.3^{\mathrm{a}}(0.3)$ & $35.8^{\mathrm{abc}}(12.0)$ & $239.2^{\mathrm{abc}}(60.7)$ & & \\
\hline Reference Flow & $103.8^{\text {cdef }}(10.1)$ & $5.8^{\mathrm{de}}(0.3)$ & $40.1^{\mathrm{bc}}(9.1)$ & $299.0^{\mathrm{c}}(23.0)$ & & \\
\hline Revolution & $92.0^{\text {bcde }}(5.0)$ & $4.0^{\mathrm{b}}(0.4)$ & $28.4^{\mathrm{ab}}(6.0)$ & $253.9^{\mathrm{abc}}(14.3$ & & \\
\hline Grandio Flow & $85.2^{\mathrm{abcd}}(23.1)$ & $4.1^{\mathrm{b}}(0.6)$ & $44.6^{\mathrm{cd}}(4.4)$ & $233.1^{\mathrm{ab}}(16.3)$ & & \\
\hline Definite Flow & $81.7^{\mathrm{abcd}}(31.4)$ & $4.3^{\mathrm{bc}}(0.7)$ & $33.4^{\mathrm{abc}}(9.1)$ & $250.3^{\mathrm{abc}}(49.3)$ & & \\
\hline Palfique Estelite High Flow & $69.3^{\mathrm{ab}}(10.3)$ & $1.6^{\mathrm{a}}(0.4)$ & $38.7^{\mathrm{abc}}(12.1)$ & $285.5^{\mathrm{bc}}(42.9)$ & & \\
\hline Admira Flow & $62.8^{\mathrm{a}}(11.0)$ & $5.4^{\mathrm{cde}}(1.4)$ & $31.2^{\mathrm{ab}}(7.2)$ & $277.3^{\mathrm{bc}}(19.2)$ & & \\
\hline \multicolumn{7}{|l|}{ Flowable compomeres } \\
\hline Compoglass Flow & $120.4^{\mathrm{fg}}(10.0)$ & $5.5^{\text {cde }}(0.3)$ & $39.6^{\mathrm{bc}}(7.2)$ & $262.2^{\mathrm{bc}}(38.4)$ & & \\
\hline PrimaFlow & $95.7^{\text {bcdef }}(11.8)$ & $4.5^{\mathrm{bc}}(0.2)$ & $38.9^{\mathrm{abc}}(7.2)$ & $195.1^{\mathrm{a}}(10.5)$ & & \\
\hline Estelite Flow Quick & $77.5^{\mathrm{abc}}(16.0)$ & $2.5^{\mathrm{a}}(0.3)$ & $26.8^{\mathrm{a}}(5.0)$ & $261.7^{\mathrm{bc}}(45.2)$ & & \\
\hline Dyract Flow & $62.9^{\mathrm{a}}(14.7)$ & $4.3^{\mathrm{bc}}(0.3)$ & $28.7^{\mathrm{ab}}(4.7)$ & $232.2^{\mathrm{ab}}(29.9)$ & & \\
\hline Material type & & & & & Vol. \% & Wt. \% \\
\hline Hybrid Composites & $116.6^{\mathrm{d}}(23.9)$ & $7.3^{\mathrm{b}}(2.3)$ & $32.5^{\mathrm{b}}(8.0)$ & $211.5^{\mathrm{a}}(71.1)$ & $60.5^{\mathrm{de}}(5.7)$ & $79.0^{\mathrm{ef}}(3.2)$ \\
\hline Packable Composites & $105.9^{\mathrm{cd}}(28.1)$ & $8.4^{\mathrm{bc}}(2.5)$ & $34.3^{\mathrm{bc}}(8.8)$ & $217.4^{\mathrm{a}}(66.5)$ & $63.0^{\mathrm{c}}(3.2)$ & $80.3^{\mathrm{f}}(3.5)$ \\
\hline Ormocer-based Composites & $104.3^{\mathrm{bcd}}(21.6)$ & $7.5^{\mathrm{b}}(2.4)$ & $35.2^{\mathrm{bc}}(9.5)$ & $216.0^{\mathrm{a}}(31.5)$ & $60.6^{\mathrm{de}}(0.4)$ & $77.0^{\mathrm{d}}(0)$ \\
\hline Nano-hybrid Composites & $103.1^{\mathrm{bcd}}(19)$ & $5.0^{\mathrm{a}}(0.8)$ & $40.5^{\mathrm{d}}(8.3)$ & $210.8^{\mathrm{a}}(63.3)$ & $59.7^{\mathrm{d}}(3.9)$ & $78.4^{\mathrm{de}}(2.5)$ \\
\hline Compomers & $94.7^{\mathrm{bc}}(34)$ & $9.2^{\mathrm{c}}(1.9)$ & $33.9^{\mathrm{bc}}(9.4)$ & $230.9^{\mathrm{ab}}(35.3)$ & $56.0^{\mathrm{c}}(6.8)$ & $74.9^{\mathrm{c}}(5.6)$ \\
\hline Flowable Compomers & $89.1^{\mathrm{ab}}(25.2)$ & $4.2^{\mathrm{a}}(1.1)$ & $33.5^{\mathrm{bc}}(8.3)$ & $237.8^{\mathrm{ab}}(42.4)$ & $43.3^{\mathrm{a}}(4.3)$ & $64.4^{\mathrm{b}}(4.1)$ \\
\hline Flowable Composites & $99.8^{\mathrm{bc}}(27.4)$ & $4.4^{\mathrm{a}}(1.6)$ & $38.3^{\mathrm{cd}}(10)$ & $264.2^{\mathrm{b}}(41.5)$ & $48.4^{\mathrm{b}}(6.5)$ & $66.1^{\mathrm{b}}(4.3)$ \\
\hline Microfilled Composites & $73.5^{\mathrm{a}}(10.9)$ & $3.8^{\mathrm{a}}(0.8)$ & $24.2^{\mathrm{a}}(3.8)$ & $246.9^{\mathrm{ab}}(37.8)$ & $42.7^{\mathrm{a}}(3.0)$ & $60.7^{\mathrm{a}}(4.0)$ \\
\hline
\end{tabular}

Data are arranged in descending order of the flexural strength value

Superscript letters indicate statistically homogeneous subgroups within a material category (Tukey's HSD test, $\alpha=0.05$ ) 